\title{
ARTICLE \\ Salivary kynurenic acid response to psychological stress: inverse relationship to cortical glutamate in schizophrenia
}

Joshua Chiappelli ${ }^{1}$, Laura M. Rowland ${ }^{1}$, Francesca M. Notarangelo ${ }^{1}$, S. Andrea Wijtenburg ${ }^{1}$, Marian A. R. Thomas ${ }^{1}$, Ana Pocivavsek ${ }^{1}$, Aaron Jones ${ }^{1}$, Krista Wisner ${ }^{1}$, Peter Kochunov ${ }^{1}$, Robert Schwarcz ${ }^{1}$ and L. Elliot Hong ${ }^{1}$

Frontal glutamatergic synapses are thought to be critical for adaptive, long-term stress responses. Prefrontal cortices, including the anterior cingulate cortex (ACC) contribute to stress perception and regulation, and are involved in top-down regulation of peripheral glucocorticoid and inflammatory responses to stress. Levels of kynurenic acid (KYNA) in saliva increase in response to psychological stress, and this stress-induced effect may be abnormal in people with schizophrenia. Here we test the hypothesis that ACC glutamatergic functioning may contribute to the stress-induced salivary KYNA response in schizophrenia. In 56 patients with schizophrenia and 58 healthy controls, our results confirm that levels of KYNA in saliva increase following psychological stress. The magnitude of the effect correlated negatively with proton magnetic resonance spectroscopy (MRS) glutamate + glutamine $(r=$ $-.31, p=.017)$ and glutamate $(r=-0.27, p=.047)$ levels in the ACC in patients but not in the controls (all $p \geq .45)$. Although, a causal relationship cannot be ascertained in this cross-sectional study, these findings suggest a potentially meaningful link between central glutamate levels and kynurenine pathway response to stress in individuals with schizophrenia.

Neuropsychopharmacology (2018) 43:1706-1711; https://doi.org/10.1038/s41386-018-0072-2

\section{INTRODUCTION}

Adaptive responses to stress involve top-down evaluation of stressful situations, optimization of mental and physiological responses, and the homeostatic resolution of these responses. The anterior cingulate cortex (ACC) is a part of the prefrontal areas involved in processing stressful or threatening stimuli and regulating emotional and behavioral responses [1, 2]. The cortical top-down regulation of emotional and behavioral stress responses involves prefrontal glutamatergic projections to the amygdala, the ventral tegmental area, and hippocampus, which are all known to causally modulate normal and abnormal stress responses [3-6]. As schizophrenia is associated with disrupted glutamatergic functions $[7,8]$ and stress is thought to play an important role in the etiology and exacerbation of the disease $[9,10]$, we hypothesize that ACC glutamatergic dysfunction may contribute to abnormal stress responses in schizophrenia.

One component of the stress response that may be particularly relevant in schizophrenia is the activation of the kynurenine pathway of tryptophan degradation. Several metabolites of this pathway are neuroactive, including kynurenic acid (KYNA), which can act as an antagonist at NMDA and a7 nicotinic receptors [11]. These effects, which are responsible for KYNA's ability to control extracellular glutamate levels in the brain (see ref. [12], for review), could contribute to cognitive deficits observed in schizophrenia [13-15]. Notably, increased levels of kynurenine and KYNA have been found in postmortem brain tissue [16, 17] and in cerebrospinal fluid (CSF) of individuals with schizophrenia [18, 19].

We previously reported that salivary KYNA rises in response to a psychological stress paradigm, with some schizophrenia patients exhibiting unusually high levels [20], although the underlying mechanism was not identified. Forebrain glutamatergic synapses are critical for regulating the hypothalamic-pituitary-adrenal (HPA) axis [4], and stress is associated with a long-lasting potentiation of glutamatergic transmission in frontal pyramidal neurons, mediated by glucocorticoid receptor activation [21]. Therefore, in the current study, our aim was to examine if levels of glutamate in the ACC, as measured with proton magnetic resonance spectroscopy (MRS), are related to the KYNA response to stress. Although, glutamate measured with MRS does not distinguish between synaptic glutamatergic activity and metabolic processes involving this amino acid, the levels may serve as a proxy imaging biomarker of glutamatergic cortical excitability [22].

\section{METHODS}

Participants

Individuals with schizophrenia spectrum disorder ( $n=56$, including 16 with schizoaffective disorder) were recruited from the outpatient clinics at the Maryland Psychiatric Research Center and neighboring mental health clinics. Healthy controls $(n=59)$ were recruited through media advertisements. Diagnoses were confirmed with the Structured Clinical Interview (SCID) for DSM-IV in all participants. Exclusion criteria included history of major neurological and medical conditions, epilepsy, cerebrovascular accident, head injury with cognitive sequelae, and mental retardation. Individuals were not excluded if they had common medical illness, such as hypertension, provided this condition was stable. Participants with an active autoimmune or infectious disorder, or who were being actively treated for such conditions, were excluded. DSM-IV interviews were used to systematically identify and exclude patients and controls with substance use disorder (except nicotine). In addition, urine spot screens were

\footnotetext{
${ }^{1}$ Department of Psychiatry, Maryland Psychiatric Research Center, University of Maryland School of Medicine, Baltimore, MD, USA
}

Correspondence: Joshua Chiappelli (jchiappe@som.umaryland.edu)

Received: 3 January 2018 Revised: 4 April 2018 Accepted: 9 April 2018

Published online: 18 April 2018 
used whenever there was suspicion of recent drug use by a participant. Controls had no current DSM-IV Axis I diagnoses and no family history of psychosis in the prior two generations. Except for one medication-free participant, all schizophrenia patients were on antipsychotic medications, including 46 taking atypical antipsychotics, five taking typical antipsychotics, and four taking a combination of antipsychotic types. The average chlorpromazine (CPZ) equivalent dosage of antipsychotic medication was $466 \pm$ 395 (standard deviation). Participants gave written informed consent. This study was approved by the University of Maryland Baltimore IRB.

\section{Clinical assessments}

Overall psychiatric symptoms were assessed by the 20-item Brief Psychiatric Rating Scale (BPRS), and negative symptoms were rated using the Brief Negative Symptom Scale (BNSS; [23]). To assess cognitive deficits, participants were tested with the Digit Symbol Coding task of the WAIS-3 and the Digit Sequencing task from the Brief Assessment of Cognition in Schizophrenia [24, 25], to measure processing speed and working memory, respectively. Deficits in these measures are among the most robust cognitive impairments in schizophrenia [26, 27].

\section{Psychological stress challenge}

The behavioral paradigm used to induce stress in participants involved two computerized tasks, the Paced Auditory Serial Addition Task (PASAT) [28] and the Mirror-Tracing Persistence Task (MTPT) [29]. The PASAT requires participants to add numbers presented on the screen consecutively; they must remember the previous number presented in order to make the correct calculation. The MTPT requires participants to guide a cursor along the outline of a star, with the movement of the cursor opposite to the movement of the mouse. In a practice session, the computer program recorded the participants' performance; this performance was used to titrate the computer program in the testing session such that the tasks were extremely difficult regardless of individual competence, with obnoxious noises played any time the participant made a mistake. The order of tasks was randomized across participants. Participants were instructed that they could quit the tasks at any time, though in doing so they would forego monetary bonus for completion; if participants quit both tasks early, they were designated "distress intolerant" (DI). Further details on the stressor employed in this study have been published elsewhere [20]. However, there is no overlap in salivary KYNA data between the current study and our previous reports $[20,30]$. The stress challenge and MRS were completed on separate days, with stress challenge generally completed first soon after study enrollment, followed by the MRS with an average time interval of 38 days. The interval was used as a covariate.

Saliva collection and biochemical assay

Saliva was collected by passive drool at four time pointsbaseline, immediately following the behavioral challenge $(0 \mathrm{~min}$ post-task), and at 20 and $40 \mathrm{~min}$ post-task. During the post-stress period, all participants sat quietly in the same room and chair alone and read magazines. All testing sessions were held between 1200 and $1600 \mathrm{~h}$. Participants were asked to refrain from eating, drinking or smoking for one hour before the testing session, and were instructed to rinse their mouths with water about 10 min prior to first saliva collection. Saliva samples were immediately stored at $-80^{\circ} \mathrm{C}$ until assay. Prior to assays, samples were thawed and centrifuged at $10,000 \times g$ for $10 \mathrm{~min}$. For KYNA measurement, $100 \mu \mathrm{l}$ of saliva were acidified with $25 \mu \mathrm{l}$ of $25 \%$ perchloric acid. After centrifugation $(16,000 \times g, 10 \mathrm{~min}), 20 \mu \mathrm{l}$ of the supernatant were subjected to high-performance liquid chromatography, and KYNA was isocratically eluted and fluorimetrically detected as previously described [31, 32]. All assays were performed blinded to group or behavior information until after the results were finalized.

\section{MRS}

All imaging was acquired using a Siemens 3T TRIO MRI (Erlangen, Germany) system equipped with a 32-channel phase array head coil. A $40 \times 30 \times 20 \mathrm{~mm}$ spectroscopic voxel was prescribed on the midsagittal slice and positioned parallel to the genu of the corpus callosum and scalp with the midline of the voxel corresponding to the middle of the genu of the corpus callosum (Fig. 1a). Spectra were acquired using very short TE (VTE) phase rotation (PR) STEAM sequence: $\mathrm{TR} / \mathrm{TM} / \mathrm{TE}=2000 / 10 / 6.5-\mathrm{ms}, \mathrm{NEX}=128,2.5-\mathrm{kHz}$ spectral width, 2048 complex points, and phases: $\varphi 1=135^{\circ}, \varphi 2=$ $22.5^{\circ}, \varphi 13=112.5^{\circ}$, and $\varphi A D C=0^{\circ}[33,34]$. A water reference $(\mathrm{NEX}=16)$ was also acquired for phase and eddy current correction, as well as quantification. A basis set of 19 metabolites was simulated using the GAVA software package [35]. The basis set was imported into LCModel (6.3-0I) and used for quantification [36]. Metabolites were corrected for the proportion of the gray matter, white matter, and CSF within each spectroscopic voxel was performed using SPM8 and in-house Matlab code [37]. Only metabolites with percent standard deviation (\%SD) of $<20 \%$ were
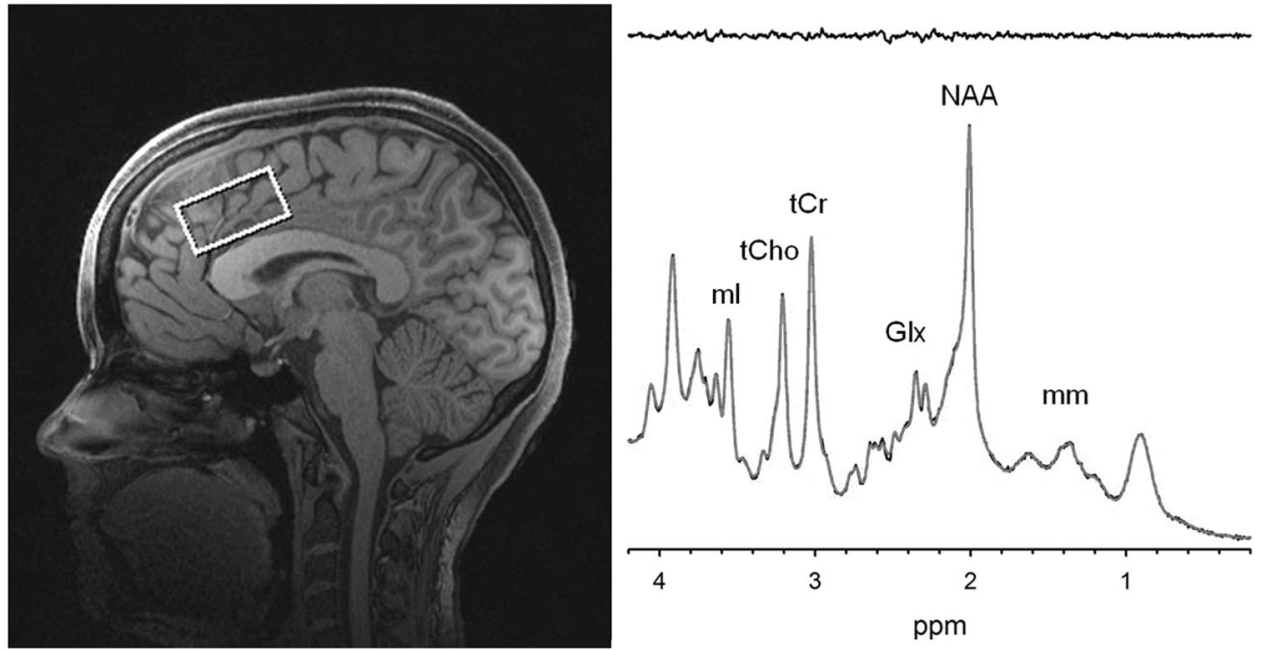

Fig. 1 Voxel placement in anterior cingulate cortex (ACC) and representative spectrum and LCModel metabolite fit (red), with residual shown in gray above 
Table 1. Summary of demographic characteristics and plasma measures

\begin{tabular}{|c|c|c|c|c|}
\hline & $\begin{array}{l}\text { Control }(n \\
=59)\end{array}$ & $\begin{array}{l}\text { Schizophrenia ( } n \\
=56 \text { ) }\end{array}$ & $\begin{array}{l}\text { Test- } \\
\text { statistic }\end{array}$ & $p$-value \\
\hline Age (years) & $33.5 \pm 13.7$ & $37.0 \pm 13.8$ & $t=1.37$ & .17 \\
\hline $\begin{array}{l}\text { Smoker/ } \\
\text { nonsmoker }\end{array}$ & $18 / 41$ & $26 / 30$ & $x^{2}=3.08$ & .08 \\
\hline Male/female & $32 / 27$ & $39 / 17$ & $x^{2}=2.89$ & .09 \\
\hline Body mass index & $26.8 \pm 6.1$ & $27.5 \pm 6.7$ & $t=0.54$ & .59 \\
\hline $\begin{array}{l}\text { Duration of } \\
\text { illness (years) }\end{array}$ & $\mathrm{n} / \mathrm{a}$ & $13.7 \pm 11.6$ & $\mathrm{n} / \mathrm{a}$ & \\
\hline $\begin{array}{l}\text { Distress } \\
\text { intolerant (n) }\end{array}$ & 12 & 19 & $\chi^{2}=2.72$ & .10 \\
\hline Glutamate & $9.0 \pm 1.2$ & $8.3 \pm 1.3$ & $F=8.56^{\mathrm{a}}$ & .004 \\
\hline $\begin{array}{l}\text { Glx (glutamate } \\
\text { + glutamine) }\end{array}$ & $11.5 \pm 1.4$ & $10.9 \pm 1.7$ & $F=3.27^{\mathrm{a}}$ & .073 \\
\hline
\end{tabular}

included in statistical analyses. Spectra with LCModel reported linewidths $>0.1 \mathrm{~Hz}$ and signal-to-noise ratio (SNR) $<10$ were excluded. Metabolite levels are reported in institutional units (i. u.). Previously, we have shown that the VTE PR-STEAM method produces excellent reproducibility as determined by a test-retest study [34]. The mean ( \pm standard deviation) SNR was $80.7 \pm 12.1$ for controls and $74.2 \pm 16.0$ for patients. The full width half maximum for controls was $0.035 \pm 0.011$ and for patients was $0.038 \pm 0.012$. The two primary measures were glutamate + glutamine $(\mathrm{Glx})$ and glutamate. The \%SD, measures of goodness of fit for metabolites, were as follows: Glx: $3.4 \pm 0.77$ vs. $3.9 \pm 1.0$ (control vs. patient, respectively-same below; $t=2.67, p=.009$ ); glutamate: $3.1 \pm 0.78$ vs. $3.4 \pm 0.87(t=2.57, p=.011)$. Proportion of gray matter in the spectroscopic voxel did not significantly differ between patients and controls $(t=1.49, p=.14)$. MR spectroscopy data for 37 patients and 28 controls were included in previous reports $[38,39]$ but none of the stress-induced KYNA data were previously reported.

\section{Statistical analyses}

Differences in demographic variables between patients and controls were analyzed with chi-square or $t$-tests, as appropriate. Univariate ANOVA were used to examine group differences in levels of glutamate, Glx, and baseline salivary KYNA, using age and sex as covariates. Repeated measures ANOVA with GreenhouseGeisser corrected statistics were employed to examine changes in KYNA across the four time points of saliva collection, with diagnosis as a between-subjects variable. KYNA responses were assessed by area under the curve of the increases (AUCi) in KYNA using the trapezoid method [40]. This measure represents the overall magnitude of the KYNA response to stress. The Pearson's correlation coefficients were used to examine the relationship between KYNA AUCi and cortical Glx and glutamate levels in patient and control samples independently. Exploratory analyses of relationships between KYNA AUCi and clinical variables were performed with the Pearson's correlation coefficients. All tests were two-tailed with $p<.05$ considered significant.

\section{RESULTS}

Group differences

Patient and control demographics are shown in Table 1. ACC glutamate levels were lower in patients $(8.3 \pm 1.3)$ than in controls $(9.0 \pm 1.2 ; F(1111)=8.56, p=.004$, corrected for age and sex). Glx

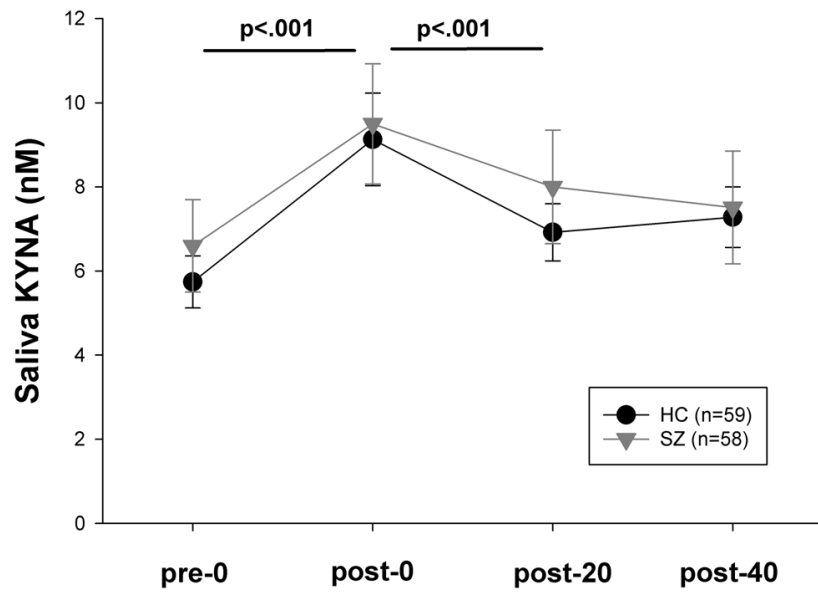

Fig. 2 Mean levels of salivary KYNA ( \pm standard deviation) prior to (pre-0), immediately after (post-0), and at 20 and 40 min following psychological stressor. The duration of the stress challenge (between pre-0 to post-0) was about 20 min

levels were not significantly different in patients $(10.9 \pm 1.7)$ compared to controls $(11.5 \pm 1.4 ; F(1111)=3.27, p=.07$, controlled for age and sex). Patients were more likely to be DI than controls, but this was not statistically significant $\left(x^{2}=2.72, p\right.$ $=.099$ ). Similar to our prior results [20], we found a trend for DI patients to have higher baseline salivary KYNA levels ( $n=19,8.47$ \pm 9.2 ) than non-DI patients ( $n=37,6.64 \pm 9.6)$, although this was not statistically significant in the current sample (Mann-Whitney $U$-test: $Z=1.49, p=.14)$; the patient sample was therefore combined in subsequent analyses. Baseline saliva KYNA levels were not significantly different between patients $(7.16 \pm 9.4)$ and controls (5.81 $\pm 4.8 ; p=.49$, correcting for age and sex).

\section{Salivary KYNA response to stress}

Repeated measures ANOVA of saliva KYNA levels showed a significant effect of time $(F(2.4,266.8)=18.3, p<.001)$, with KYNA levels immediately after the psychological stress challenge (at the post- 0 time-point) being significantly greater than at baseline ( $p$ $<.001)$ or $20 \mathrm{~min}$ post-stress $(p<.001)$ (Fig. 2$)$. However, there were no main effect of diagnosis $(F(1,111)=0.22, p=0.64)$ or time $\times$ diagnosis effect $(F=0.40, p=0.71)$, suggesting a robust KYNA response to this psychological stressor in the entire sample, but with no clear difference in stress response between groups in this sample.

Relationship of KYNA response to stress with neurochemistry In the patients, the salivary KYNA response as measured by $\mathrm{AUCi}$ was significantly correlated with Glx $(r=-.32, p=.017)$ and glutamate $(r=-.27, p=.047)$ (Fig. 3 and Table 2). These correlations were nonsignificant in controls.

Regression models were used to examine if the correlations between glutamate or Glx and KYNA AUCi observed in patients were attenuated when age, sex, and smoking status were accounted for. Glx remained a significant predictor of saliva KYNA AUCi in this model $(\beta=-.30, p=.033)$, though the relationship of glutamate to KYNA AUCi was attenuated $(\beta=-.26, p=.085)$. When time interval between stress challenge and MRS is added as a covariate, Glx remains a significant predictor of KYNA AUCi $(\beta=-.30, p=.035, p$ $=.023)$, as does glutamate $(\beta=-.33, p=.049)$.

Relationship of salivary KYNA response to clinical factors Antipsychotic medication use, measured as CPZ equivalent dosage, was not significantly correlated with KYNA AUCi (rho = $-.12, p=.41$ ), Glx (rho $=-.13, p=.37$ ) or glutamate (rho $=-.14$, $p=.34$ ) levels in patients. Body mass index was also not 

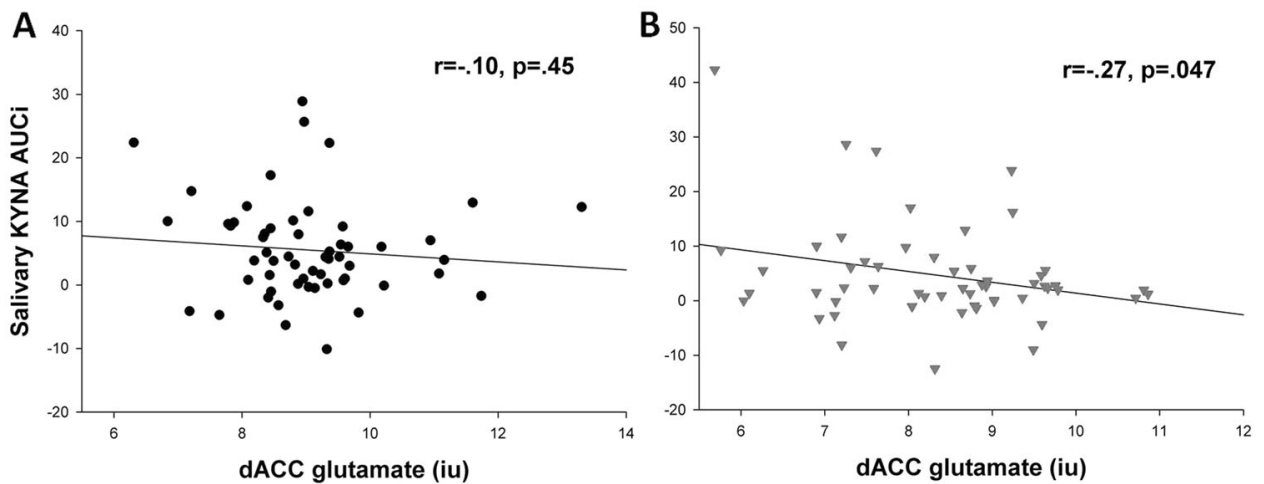

Fig. 3 Scatterplot of salivary kynurenic acid (KYNA) response to psychological stress and glutamate levels in dorsal anterior cingulate cortex (dACC), reported in institutional units (i.u.), for a healthy controls and $\mathbf{b}$ schizophrenia patients. AUCi area under the curve in regard to increase

Table 2. Pearson's correlation coefficients for the relationship between the KYNA response to stress (salivary KYNA AUCi) and metabolite concentrations in the ACC, measured by MRS

\begin{tabular}{lll}
\hline Metabolite & Controls $(n=58)$ & Schizophrenia $(n=55)$ \\
\hline Glutamate & $r=-.10, p=.45$ & $r=-.27, p=.047$ \\
Glx & $r=-.05, p=.71$ & $r=-.32, p=.017$ \\
\hline
\end{tabular}

significantly correlated with KYNA AUCi in either controls $(p=.36)$ or patients $(p=.18)$, controlling for age. Among patients, there were no significant correlations between salivary KYNA AUCi and cognitive measures (working memory and processing speed) or with symptom ratings (BPRS and BNSS scores) (all $p>.05$, uncorrected). Levels of glutamate and Glx were not correlated with BPRS or BNSS scores (all $p>.05$ ); there was a nominally significant correlation between glutamate and processing speed score $(r=.31, p=.031)$, though this was not significant after Bonferroni correction for multiple comparisons. Duration of illness negatively predicted glutamate levels after covarying for age and sex $(\beta=-.81, p=.020)$, but did not predict salivary KYNA AUCi $(p=.98)$.

\section{DISCUSSION}

The present study revealed that levels of salivary KYNA were significantly increased following exposure to a laboratory psychological stressor in both patients and controls. In patients, lower glutamate levels in the ACC were associated with a higher KYNA response to the psychological stress, but this relationship was not significant in the controls. These findings indicate that the relationship of these two metabolites may be of pathophysiological significance in schizophrenia.

The medial prefrontal cortex (mPFC) and the ACC play important roles in coordinating the response to stress through glutamatergic signaling to the subcortical regions, including the amygdala, the ventral tegmental area, and the hippocampus $[3,6]$. This frontal-limbic network regulates glucocorticoid production via the HPA, and also influences the immune system via adrenergic signaling and the cholinergic anti-inflammatory pathway $[41,42]$. In turn, cortisol induces the activity of tryptophan 2,3dioxygenase (TDO) [43]; and adrenergic activity also modulates the production of interferon gamma, a main determinant of indoleamine 2,3-dioxygenase activation (IDO) [44, 45]. TDO and IDO are the main enzymes responsible for the oxidative ring opening of the essential amino acid tryptophan, initiating the kynurenine pathway and resulting in the neosynthesis of KYNA and other kynurenine pathway metabolites downstream [46]. In designing the present study, we had therefore hypothesized that abnormally reduced glutamatergic signaling in the ACC may secondarily lead to a heightened salivary KYNA response to stress in schizophrenia. However, though our findings are consistent with this idea, our cross-sectional study design did not allow us to determine the direction of the hypothesized causal relationship(s) between glutamate levels, determined by MRS, and salivary KYNA concentrations. In other words, the observed stress-induced increase in KYNA levels in the saliva may conceivably also be the source, rather than the consequence, of the abnormal glutamate levels that we detected in the brain of individuals with schizophrenia.

Reciprocal, inverse interactions between glutamate and KYNA have indeed been consistently demonstrated in the rodent brain $[47,48]$ (see ref. [12], for review) though they have so far not been carefully investigated in humans. In rodents, peripheral administration of kynurenine or intracerebral administration of KYNA results in an acute decrease in extracellular glutamate levels, as measured by in vivo microdialysis [47, 49], whereas genomic elimination [50] or pharmacological inhibition of the enzyme kynurenine aminotransferase II, which is critically involved in KYNA neosynthesis in the mammalian brain [51], has the opposite effect $[15,52,53]$. In conceptual agreement, we have previously also found an inverse correlation between the kynurenine:tryptophan ratio in blood and glutamate levels in white matter in both controls and schizophrenia patients [54]. As kynurenine, but not KYNA, readily crosses the blood-brain barrier [55], we hypothesized that the decrease in central glutamate might be initiated by a stress-induced increase in circulating kynurenine levels. As mentioned above, however, this interpretation remains tentative since the directionality of the relationship between changes in KYNA levels in the periphery and the central nervous system still requires additional evaluation.

Another limitation of the present study, as well as many other MRS-based studies measuring brain glutamate and Glx, is that the observed concentration of the amino acids cannot be interpreted solely as a reflection of synaptic glutamatergic activity, but also reflects several metabolic events, as well as the contribution of cell types that do not directly participate in excitatory neurotransmission [22]. On the other hand, resting-state levels of glutamate are associated with neural activation in behavioral tasks [56]. The present work is also limited by the fact that most of the patients in this study were chronically ill and taking antipsychotics. However, our analyses did not reveal an influence of antipsychotic medication dosage on salivary KYNA responses or MRS glutamate and Glx measures. Another obvious limitation is the inability to directly determine KYNA levels in the mPFC/ACC in humans in vivo. The results of this study encourages animal experiments to clarify the relationships between kynurenine pathway metabolites 
in saliva vs. brain on one hand, and glutamate and Glx levels in the brain. Such efforts will not only be relevant for interpreting the present findings in patients with schizophrenia, but can be expected to have significant implications for questions in kynurenine pathway neurophysiology and neuropathology in general [11].

Our earlier study using the same psychological stress paradigm [20] found salivary KYNA responses were more prominent in patients who were DI. In the current study, we found similar trends, although the $\mathrm{DI}$ sample is too small to permit reliable assessment on whether the observed glutamate vs. KYNA response relationship is influenced by different subgroup of patients. Further studies with increased sample sizes are needed to test the follow-up hypothesis that the brain glutamatergic relationship with KYNA responses to stress in schizophrenia may separate different subgroup of patients.

In summary, the data reported here raise the possibility that central glutamatergic dysfunction in schizophrenia can be meaningfully related to the dynamics of salivary KYNA response to stress. Although, the direction of causality and its precise functional significance remain to be investigated, the outcome measures described here may be useful as a biomarker in evaluating how therapeutic interventions targeting the kynurenine pathway may help patients with schizophrenia.

\section{ACKNOWLEDGEMENTS}

We sincerely thank Frank Gaston and Hongji Chen for spectral processing. The support was received from the National Institutes of Health (grant numbers K23MH112010, P50 MH103222, U01MH108148, R01MH112180, R01MH094520, R01MH096263, and T32MH067533).

\section{ADDITIONAL INFORMATION}

Competing interests: Dr. Hong has received or plans to receive research funding or consulting fees from Mitsubishi, Your Energy Systems LLC, Neuralstem, Taisho Pharmaceutical, Sound Pharma, Heptares, Pfizer, and Takeda. All other authors declare no competing interests.

Publisher's note: Springer Nature remains neutral with regard to jurisdictional claims in published maps and institutional affiliations.

\section{REFERENCES}

1. Johansen JP, Fields HL. Glutamatergic activation of anterior cingulate cortex produces an aversive teaching signal. Nat Neurosci. 2004;7:398-403.

2. Shackman AJ, Salomons TV, Slagter HA, Fox AS, Winter JJ, Davidson RJ. The integration of negative affect, pain and cognitive control in the cingulate cortex. Nat Rev Neurosci. 2011;12:154-67.

3. Geisler S, Wise RA. Functional implications of glutamatergic projections to the ventral tegmental area. Rev Neurosci. 2008;19:227-44.

4. Hartmann J, Dedic N, Pöhlmann ML, Häusl A, Karst H, Engelhardt C, et al. Forebrain glutamatergic, but not GABAergic, neurons mediate anxiogenic effects of the glucocorticoid receptor. Mol Psychiatry. 2017;22:466-75.

5. Refojo D, Schweizer M, Kuehne C, Ehrenberg S, Thoeringer C, Vogl AM, et al. Glutamatergic and dopaminergic neurons mediate anxiogenic and anxiolytic effects of CRHR1. Science. 2011;333:1903-7.

6. Wei J, Zhong P, Qin L, Tan T, Yan Z. Chemicogenetic restoration of the prefrontal cortex to amygdala pathway ameliorates stress-induced deficits. Cereb Cortex. 2017;11:1-11.

7. Javitt DC. Glutamate as a therapeutic target in psychiatric disorders. Mol Psychiatry. 2004;9:984-97.

8. Coyle JT, Tsai G, Goff DC. Ionotropic glutamate receptors as therapeutic targets in schizophrenia. Curr Drug Targets CNS Neurol Disord. 2002;1:183-9.

9. Myin-Germeys I, van Os J. Stress-reactivity in psychosis: evidence for an affective pathway to psychosis. Clin Psychol Rev. 2007;27:409-24.

10. Docherty NM, St Hilaire A, Aakre JM, Seghers JP. Life events and high-trait reactivity together predict psychotic symptom increases in schizophrenia. Schizophr Bull. 2009;35:638-45.

11. Schwarcz R, Bruno JP, Muchowski PJ, Wu HQ. Kynurenines in the mammalian brain: when physiology meets pathology. Nat Rev Neurosci. 2012;13:465-77.
12. Schwarcz R. Kynurenines and glutamate: multiple links and therapeutic implications. In: Schwarcz R, editor. Neuropsychopharmacology: a tribute to Joseph T. Coyle. Advances in Pharmacology, vol. 76. Cambridge, MA: Academic Press; 2016. p. 13-37

13. Kozak R, Campbell BM, Strick CA, Horner W, Hoffmann WE, Kiss T, et al. Reduction of brain kynurenic acid improves cognitive function. J Neurosci. 2014;34:10592-602.

14. Wonodi I, Schwarcz R. Cortical kynurenine pathway metabolism: a novel target for cognitive enhancement in schizophrenia. Schizophr Bull. 2010;36:211-8.

15. Wu HQ, Okuyama M, Kajii Y, Pocivavsek A, Bruno JP, Schwarcz R. Targeting kynurenine aminotransferase II in psychiatric diseases: promising effects of an orally active enzyme inhibitor. Schizophr Bull. 2014;40:S152-8.

16. Sathyasaikumar KV, Stachowski EK, Wonodi I, Roberts RC, Rassoulpour A, McMahon RP, et al. Impaired kynurenine pathway metabolism in the prefrontal cortex of individuals with schizophrenia. Schizophr Bull. 2011;37:1147-56.

17. Schwarcz R, Rassoulpour A, Wu HQ, Medoff D, Tamminga CA, Roberts RC. Increased cortical kynurenate content in schizophrenia. Biol Psychiatry. 2001;50:521-30.

18. Linderholm KR, Skogh E, Olsson SK, Dahl ML, Holtze M, Engberg G, et al. Increased levels of kynurenine and kynurenic acid in the CSF of patients with schizophrenia. Schizophr Bull. 2012;38:426-32.

19. Nilsson LK, Linderholm KR, Engberg G, Paulson L, Blennow K, Lindström LH, et al. Elevated levels of kynurenic acid in the cerebrospinal fluid of male patients with schizophrenia. Schizophr Res. 2005;80:315-22.

20. Chiappelli J, Pocivavsek A, Nugent KL, Notarangelo FM, Kochunov $P$, Rowland LM, et al. Stress induced kynurenic acid as a potential biomarker for schizophrenia patients with distress intolerance. JAMA Psychiatry. 2014;71:761-8.

21. Yuen EY, Liu W, Karatsoreos IN, Ren Y, Feng J, McEwen BS, et al. Mechanisms for acute stress-induced enhancement of glutamatergic transmission and working memory. Mol Psychiatry. 2011;16:156-70.

22. Wijtenburg SA, Yang S, Fischer BA, Rowland LM. In vivo assessment of neurotransmitters and modulators with magnetic resonance spectroscopy: application to schizophrenia. Neurosci Biobehav Rev. 2015;51:276-95.

23. Kirkpatrick B, Strauss GP, Nguyen L, Fischer BA, Daniel DG, Cienfuegos A, et al. The brief negative symptom scale: psychometric properties. Schizophr Bull. 2011;37:300-5. 2011

24. Keefe RS, Goldberg TE, Harvey PD, Gold JM, Poe MP, Coughenour L. The brief assessment of cognition in schizophrenia: reliability, sensitivity, and comparison with a standard neurocognitive battery. Schizophr Res. 2004;68:283-97.

25. Wechsler D. Wechsler adult intelligence scale - third edition (WAIS-III). San Antonio, TX: Harcourt Assessment; 1997.

26. Dickinson D, Ragland JD, Gold JM, Gur RC. General and specific cognitive deficits in schizophrenia: Goliath defeats David? Biol Psychiatry. 2008;64:823-7.

27. Forbes NF, Carrick LA, McIntosh AM, Lawrie SM. Working memory in schizophrenia: a meta-analysis. Psychol Med. 2009;39:889-905.

28. Lejuez C, Kahler CW, Brown RA. A modified computer version of the Paced Auditory Serial Addition Task (PASAT) as a laboratory based stressor. Behav Ther. 2003;26:290-3.

29. Strong D, Lejuez C, Daughters S, Marinello M, Kahler C, Brown R. The computerized mirror tracing task version 1. 2003. Unpublished manual.

30. Nugent KL, Chiappelli J, Rowland LM, Daughters SB, Hong LE. Distress intolerance and clinical functioning in persons with schizophrenia. Psychiatry Res. 2014;220:31-6

31. Olsson SK, Larsson MK, Erhardt S. Subchronic elevation of brain kynurenic acid augments amphetamine-induced locomotor response in mice. J Neural Transm. 2012;119:155-63.

32. Pocivavsek A, Wu HQ, Elmer Gl, Bruno JP, Schwarcz R. Pre- and postnatal exposure to kynurenine causes cognitive deficits in adulthood. Eur J Neurosci. 2012;35:1605-12.

33. Wijtenburg SA, Knight-Scott J. Very short echo time improves the precision of glutamate detection at $3 \mathrm{~T}$ in $1 \mathrm{H}$ magnetic resonance spectroscopy. J Magn Reson Imaging. 2011;34:645-52.

34. Wijtenburg SA, Gaston FE, Spieker EA, Korenic SA, Kochunov P, Hong LE, et al. Reproducibility of phase rotation STEAM at 3T: focus on glutathione. Magn Reson Med. 2014;72:603-9.

35. Soher BJ, Young K, Bernstein A, Aygula Z, Maudsley AA. GAVA: spectral simulation for in vivo MRS applications. J Magn Reson. 2007;185:291-9.

36. Provencher SW. Estimation of metabolite concentrations from localized in vivo proton NMR spectra. Magn Reson Med. 1993;30:672-9.

37. Gasparovic C, Song T, Devier D, Bockholt HJ, Caprihan A, Mullins PG, et al. Use of tissue water as a concentration reference for proton spectroscopic imaging. Magn Reson Med. 2006;55:1219-26.

38. Rowland LM, Summerfelt A, Wijtenburg SA, Du X, Chiappelli JJ, Krishna N, et al. Frontal glutamate and $\gamma$-aminobutyric acid levels and their associations with 
mismatch negativity and digit sequencing task performance in schizophrenia. JAMA Psychiatry. 2016;73:166-74.

39. Wijtenburg SA, Wright SN, Korenic SA, Gaston FE, Ndubuizu N, Chiappelli J, et al. Altered glutamate and regional cerebral blood flow levels in schizophrenia: a 1H-MRS and pCASL study. Neuropsychopharmacology. 2017;42:562-71.

40. Pruessner JC, Kirschbaum C, Meinlschmid G, Hellhammer DH. Two formulas for computation of the area under the curve represent measures of total hormone concentration versus time-dependent change. Psychoneuroendocrinology. 2003;28:916-31.

41. Marsland AL, Kuan DC, Sheu LK, Krajina K, Kraynak TE, Manuck SB, et al. Systemic inflammation and resting state connectivity of the default mode network. Brain Behav Immun. 2017;62:162-70.

42. Rosas-Ballina M, Tracey KJ. The neurology of the immune system: neural reflexes regulate immunity. Neuron. 2009;64:28-32.

43. Salter M, Pogson Cl. The role of tryptophan 2,3-dioxygenase in the hormonal control of tryptophan metabolism in isolated rat liver cells. Effects of glucocorticoids and experimental diabetes. Biochem J. 1985;229:499-504.

44. Malarkey WB, Wang J, Cheney C, Glaser R, Nagaraja H. Human lymphocyte growth hormone stimulates interferon gamma production and is inhibited by cortisol and norepinephrine. J Neuroimmunol. 2002;123:180-7.

45. Wahle M, Neumann RP, Moritz F, Krause A, Buttgereit F, Baerwald CG. Beta2adrenergic receptors mediate the differential effects of catecholamines on cytokine production of PBMC. J Interferon Cytokine Res. 2005;25:384-94.

46. Larkin PB, Sathyasaikumar KV, Notarangelo FM, Funakoshi H, Nakamura T, Schwarcz R, et al. Tryptophan 2,3-dioxygenase and indoleamine 2,3-dioxygenase 1 make separate, tissue-specific contributions to basal and inflammation-induced kynurenine pathway metabolism in mice. Biochim Biophys Acta. 2016;1860:2345-54.

47. Konradsson-Geuken A, Wu HQ, Gash CR, Alexander KS, Campbell A, Sozeri Y, et al. Cortical kynurenic acid bi-directionally modulates prefrontal glutamate levels as assessed by microdialysis and rapid electrochemistry. Neuroscience. 2010;169:1848-59.

48. Wu HQ, Ungerstedt $U$, Schwarcz R. Regulation of kynurenic acid synthesis studied by microdialysis in the dorsal hippocampus of unanesthetized rats. Eur J Pharmacol. 1992;213:375-80.

49. Wu HQ, Pereira EF, Bruno JP, Pellicciari R, Albuquerque EX, Schwarcz R. The astrocyte-derived alpha7 nicotinic receptor antagonist kynurenic acid controls extracellular glutamate levels in the prefrontal cortex. J Mol Neurosci. 2010;40:204-10.

50. Potter MC, Elmer Gl, Bergeron R, Albuquerque EX, Guidetti $P, W u H Q$, et al. Reduction of endogenous kynurenate formation enhances extracellular glutamate, hippocampal plasticity and cognitive behavior. Neuropsychopharmacology. 2010;35:1734-42.

51. Guidetti $P$, Okuno E, Schwarcz R. Characterization of rat brain kynurenine aminotransferases I and II. J Neurosci Res. 1997;50:457-65.

52. Bortz DM, Wu HQ, Schwarcz R, Bruno JP. Oral administration of a specific kynurenic acid synthesis (KAT II) inhibitor attenuates evoked glutamate release in rat prefrontal cortex. Neuropharmacology. 2017;121:69-78.

53. Koshy Cherian A, Gritton H, Johnson DE, Young D, Kozak R, Sarter M. A systemically-available kynurenine aminotransferase II (KAT II) inhibitor restores nicotine-evoked glutamatergic activity in the cortex of rats. Neuropharmacology. 2014;82:41-8.

54. Chiappelli J, Postolache T, Kochunov P, Rowland LM, Wijtenburg SA, Shukla DK, et al. Tryptophan metabolism and white matter integrity in schizophrenia. Neuropsychopharmacology. 2016;41:2587-95.

55. Fukui S, Schwarcz R, Rapoport SI, Takada Y, Smith QR. Blood-brain barrier transport of kynurenines: implications for brain synthesis and metabolism. J Neurochem. 1991;56:2007-17.

56. Falkenberg LE, Westerhausen R, Specht K, Hugdahl K. Resting-state glutamate level in the anterior cingulate predicts blood-oxygen level-dependent response to cognitive control. Proc Natl Acad Sci USA. 2012;109:5069-73. 